\title{
Óbitos infantis em um estado do Nordeste brasileiro: características e evitabilidade.
}

\author{
Infant deaths in a State of Brazilian Northeast: \\ features and avoidability.
}

\section{Las muertes infantiles en un estado en el noreste de Brasil: características y evitables.}

Jorge Otávio Maia BARRETO ${ }^{1}$

Inez Sampaio NERY ${ }^{2}$

\begin{abstract}
RESUMO: Objetivo: analisar características e evitabilidade dos óbitos infantis do Piauí de 2006 a 2011. Métodos: A partir da vinculação dos dados oficiais sobre nascimentos e óbitos foram analisadas variáveis de escolaridade e raça/cor da mãe, peso ao nascer, número de consultas de pré-natal e evitabilidade do óbito infantil. Resultados: a amostra foi constituída por 2.584 óbitos infantis, com 7,7\% de lacunas de informação nas variáveis selecionadas. A mortalidade infantil no Piauí foi predominante entre mães pardas, com menor escolaridade, menos consultas de pré-natal e entre crianças com peso ao nascer inferior a 2.500 gramas. Verificou-se que $72,4 \%$ dos óbitos eram evitáveis, especialmente pela adequada atenção à gestante, ao parto e neonato. Conclusão: persistiu elevado percentual de mortes infantis preveníveis no Piauí. O monitoramento conjunto dos dados sobre mortalidade e nascimento é relevante para a avaliação e planejamento para reduzir a mortalidade infantil evitável.
\end{abstract}

Palavras-chave: Política de Saúde, Mortalidade Infantil, Causas de Morte, Equidade em Saúde, Epidemiologia Descritiva

\begin{abstract}
Objective: to analyse characteristics and avoidability of infant deaths of Piauí from 2006 to 2011. Methods: we linked the official data on births and deaths to analyze variables of schooling and race / skin color of mother, birth weight, number of antenatal visits and preventability of infant death. Results: the sample was consisted of 2,584 infant deaths, with $7.7 \%$ of information gaps in the selected variables. Infant mortality in Piauí was prevalent among brown mothers with less education, less prenatal consultations and between children with birth weight less than 2,500 grams. $72.4 \%$ of the deaths were considered preventable, especially by the adequate care for pregnant women, delivery labor and the newborn. Conclusion: high rates of preventable child

1 Doutor em Políticas Públicas, Mestrado em Políticas Públicas em Saúde, Fundação Oswaldo Cruz, Brasil. SQN 210, Bloco G, Apto. 507, Asa Norte, Brasília, Distrito Federal, Brasil, CEP 70862-070, Telefone: 61-99111606; Email: jorgeomaia@hotmail.com.

2 Doutora em Enfermagem, Programa de Pós-graduação em Políticas Públicas e Mestrado em Enfermagem, Universidade Federal do Piauí, Brasil. Universidade Federal do Piauí - UFPI, Campus Universitário Ministro Petrônio Portella, Bairro Ininga, Teresina, PI, Brasil, CEP 64049-550, Telefone: 86-3215.1103; Email: ineznery.ufpi@gmail.com
\end{abstract}


deaths in Piaui was maintained. The joint monitoring of data on mortality and birth is relevant to the assessment and planning to reduce preventable child mortality.

Keywords: Health Policy, Infant Mortality, Cause of Death, Equity in Health, Descriptive Epidemiology

\section{INTRODUÇÃO}

A mortalidade infantil é um indicador de desenvolvimento social utilizado em todo mundo para evidenciar desigualdades sociais e iniquidades em saúde, sendo sua redução um dos Objetivos de Desenvolvimento do Milênio pelo programa das Nações Unidas para países em desenvolvimento ${ }^{1}$. O óbito infantil reflete um problema complexo que inclui determinantes socioeconômicos, mas também fatores relacionados com o acesso e qualidade dos serviços de saúde ${ }^{2}$. Nesse contexto, as informações sobre o óbito infantil, qualidade da assistência em saúde e condições socioeconômicas dos sujeitos afetados por este problema são imprescindíveis para seu enfrentamento.

A redução das mortes infantis decorrentes de falhas na atenção à saúde requer informações que apoiem a identificação de problemas e suas potenciais causas. No Brasil, adotou-se uma lista de mortes evitáveis por intervenções no âmbito do sistema de saúde para o grupo etário menor de 5 $\operatorname{anos}^{3-4}$. A Lista Brasileira de Mortes Evitáveis classificou os óbitos potencialmente reduzíveis por grupos de ações de saúde, incluindo: i) ações de imunoprevenção; ii) atenção à mulher na gestação, parto, feto e ao recém-nascido; iii) ações de diagnóstico e tratamento e iv) ações de promoção à saúde, vinculadas a ações de atenção à saúde ${ }^{3-4}$.

O Ministério da Saúde, responsável pela coordenação nacional dos esforços para a redução da mortalidade infantil no Sistema Único de Saúde (SUS), preconiza a incorporação no cotidiano das secretarias de saúde a produção de dados e sua sistematização, através dos sistemas oficiais de informação sobre eventos vitais ${ }^{5}$, a saber, o Sistema de Informação sobre Nascidos Vivos (SINASC) $)^{6}$ e Sistema de Informação sobre Mortalidade (SIM) ${ }^{7}$.

No Nordeste do Brasil, os fatores de risco para mortalidade infantil estão relacionados com a baixa idade e escolaridade das mães, baixo peso ao nascer, prematuridade, gravidez múltipla e parto cesáreo, remetendo à necessidade de considerar-se a conjuntura social e cultural onde se inserem as gestantes, a fim viabilizar ações de promoção e prevenção para a saúde materna e infantil ${ }^{8}$. Por outro lado, também se reconhece a necessidade de que a informação seja avaliada quanto à sua qualidade e consistência, inclusive para fins de correção que possibilite melhor estimativa do risco de morte infantil entre as populações mais vulneráveis 9 .

O Estado do Piauí apresentou redução da taxa de mortalidade infantil média nos últimos dez anos, mas se manteve entre as mais elevadas no país, com 20,8 óbitos infantis por mil nascidos vivos, em $2011^{10}$. Apesar dos importantes avanços na qualidade da informação do estado ${ }^{11}$, monitoramento e avaliação contínuos dos registros de nascimentos e óbitos são necessários para apoiar a tomada de 
decisão na política de saúde, especialmente se consideradas as necessidades de contribuição par a melhoria da informação em âmbito nacional ${ }^{12}$.

O objetivo deste estudo foi analisar características da mortalidade infantil do Estado do Piauí, no período de 2006 a 2011, a partir dos dados oficiais do SIM e SINASC, considerando aspectos socioeconômicos, o acesso aos serviços de saúde e a evitabilidade do óbito. Os resultados poderão subsidiar a discussão sobre a mortalidade infantil na região e no Brasil, favorecendo a efetividade das políticas públicas endereçadas ao problema.

\section{METODOLOGIA}

Trata-se de estudo descritivo visando à caracterização dos óbitos infantis ocorridos na população residente do Piauí no período de 2006 a 2011, considerando o perfil socioeconômico da população afetada, o acesso ao acompanhamento pré-natal e a evitabilidade potencial destas mortes.

Para a constituição da amostra, os dados sobre os óbitos infantis, disponíveis nas bases do SIM, foram vinculados aos do SINASC, mediante técnica de linkage, adotando-se como chave primária o número da Declaração de Nascido Vivo (DNV). O linkage tem se mostrado útil à investigação de fatores relacionados à mortalidade infantil e ganhado relevância como técnica auxiliar ao estudo de problemas de saúde pública ${ }^{13}$. Os registros de óbito infantil que não continham a informação da DNV foram excluídos. Também se analisaram as lacunas de informação das variáveis de interesse após o linkage, em comparação às bases originais (SIM/SINASC), calculando-se os percentuais de falhas de registro por variável.

Utilizou-se estatística descritiva para a determinação da frequência e distribuição na amostra das características a seguir descritas, considerando-se as informações e parâmetros disponíveis no SINASC:

i) Escolaridade da mãe em faixas de anos de estudo: nenhuma; de 1 a 4; de 5 a 8; de 9 a 11; 12 ou mais;

ii) Raça/cor da mãe: Branca; Preta; Amarela; Parda e Indígena;

iii) Peso ao nascer em gramas;

iv) Faixas de consultas de pré-natal: nenhuma; 1 a 3; 4 a $6 ; 7$ e mais.

Quanto à evitabilidade dos óbitos, foi considerada a causa básica registrada no SIM e a classificação da Lista Brasileira de Mortes Evitáveis para o grupo etário menor de $5 \operatorname{anos}^{3-4}$. Os óbitos infantis foram agregados em grupos de óbitos reduzíveis por ações de saúde, e calculada a distribuição na amostra, conforme segue: Grupo 1, óbitos reduzíveis por ações de imunoprevenção; Grupo 2, óbitos reduzíveis por adequada atenção à mulher na gestação; Grupo 3, óbitos reduzíveis 
por adequada atenção à mulher no parto; Grupo 4, óbitos reduzíveis por adequada atenção ao recémnascido; Grupo 5, óbitos reduzíveis por ações adequadas de diagnóstico e tratamento e Grupo 6, óbitos reduzíveis por ações adequadas de promoção à saúde, vinculadas a ações adequadas de atenção à saúde.

A coleta e compilação dos dados foi realizada de janeiro a abril de 2014, junto às bases nacionais do Ministério da Saúde, disponíveis do sítio do seu Departamento de Informática (DATASUS), e a análise foi realizada com apoio dos softwares Microsoft Excel $^{\circledR}(2010)$ e TabWin $3.6^{\odot}(2011)$.

Este estudo foi realizado com base em dados secundários de acesso aberto e não requereu submissão a comitê de ética na pesquisa, mas buscou-se seguir, quando aplicáveis, os princípios éticos e de confidencialidade recomendados pela Resolução n.466/2012 do Conselho Nacional de Saúde.

\section{RESULTADOS}

O banco de dados do SINASC apresentou 311.784 nascimentos e o SIM registrou 5.705 óbitos infantis de residentes no Piauí no período. Após a vinculação dos dados e exclusão dos registros com lacuna da chave primária (número da DNV), restaram 2.584 observações, representando $45,3 \%$ dos óbitos infantis totais registrados no SIM no período, implicando que mais da metade dos óbitos infantis não apresentavam o número da respectiva DNV.

Observou-se ao longo dos anos analisados, redução percentual mais acentuada dos óbitos infantis do que nos nascimentos. O número de nascidos vivos foi reduzido em 9,4\%, entre 2006 e 2011 (55.342-50.144), enquanto que os óbitos registrados reduziram em 21,9\%. A Taxa de Mortalidade Infantil do estado, calculada a partir destes dados, por sua vez, registrou redução de $13,8 \%$ (19,616,9) (Tabela 1).

Tabela 1 - Nascidos vivos, óbitos infantis e taxa de mortalidade infantil na população residente, Piauí, 2006-2011

\begin{tabular}{lrrrrrrr}
\hline Ano & 2006 & 2007 & 2008 & 2009 & 2010 & 2011 & Total \\
Nascidos vivos & 55.342 & 53.214 & 52.664 & 50.996 & 49.424 & 50.144 & 311.784 \\
Óbitos infantis & 1.084 & 1.056 & 972 & 913 & 833 & 847 & 5.705 \\
Taxa de Mortalidade Infantil* & 19,6 & 19,8 & 18,5 & 17,9 & 16,8 & 16,9 & 18,3 \\
\hline
\end{tabular}

Fonte: SINASC/SIM, Ministério da Saúde, 2014. * Taxa de Mortalidade Infantil por 1.000 nascidos vivos.

A tabela 2 mostra resultados da análise das lacunas de informação sobre escolaridade e raça/ cor da mãe, peso ao nascer da criança, número de consultas de pré-natal e causa básica do óbito. Identificou-se que os dados do SINASC apresentaram menos lacunas que o SIM nas variáveis selecionadas, excetuando-se o dado sobre a causa básica do óbito, presente apenas na Declaração de Óbito (DO). As informações do SIM sobre escolaridade e raça/cor da mãe apresentaram 
respectivamente 22,4 e $21,5 \%$ de falhas, enquanto as informações sobre o peso ao nascer, nas informações de mortalidade, mostraram lacuna em 13\% dos registros. Após a vinculação ainda identificou-se alguma lacuna residual em $7,7 \%$ dos registros.

Tabela 2 - Lacunas de informação variáveis selecionadas nos registros de nascimento e óbitos infantis das bases do SINASC, SIM e da amostra, após linkage, Piauí, 2006-2011

\begin{tabular}{|c|c|c|c|c|c|c|}
\hline \multirow{2}{*}{ Variáveis } & \multicolumn{2}{|c|}{ DNV (SINASC) } & \multicolumn{2}{|c|}{ DO (SIM) } & \multicolumn{2}{|c|}{ Após linkage } \\
\hline & $\mathrm{n}$ & $\%$ & $\mathrm{n}$ & $\%$ & $\mathrm{n}$ & $\%$ \\
\hline Número de DNV(SINASC)/DO(SIM) & 311.784 & - & 5.705 & - & - & - \\
\hline Escolaridade da mãe & 4.927 & $1,58 \%$ & 1.278 & $22,4 \%$ & 56 & $2,2 \%$ \\
\hline Raça/cor da mãe & 7.818 & $2,51 \%$ & 1.228 & $21,5 \%$ & 75 & $2,9 \%$ \\
\hline Peso ao nascer & 830 & $0,27 \%$ & 741 & $13 \%$ & 16 & $0,6 \%$ \\
\hline Número de consultas de pré-natal & 3.657 & $1,17 \%$ & - & - & 51 & $1,9 \%$ \\
\hline Causa Básica do óbito infantil & - & - & - & - & - & - \\
\hline
\end{tabular}

Fonte: SINASC/SIM, Ministério da Saúde, 2014.

A tabela 3 expõe os resultados da caracterização do óbito infantil, mostrando maior prevalência na faixa de escolaridade materna do ensino médio, variando positivamente de 24,2\% (2006) a 42,7\% (2011). Já a proporção de mortes infantis nas faixas das mães analfabetas variou negativamente de $5,7 \%$ a $4,1 \%$, bem como na faixa de escolaridade materna de 1-4 anos de estudo, a qual caiu de 24,6 para $11,2 \%$. Entre as mães com ensino superior, a variação foi de 8,7 para 7,6\%, no período analisado.

Para a variável raça/cor da mãe, a maior prevalência de óbitos infantis foi observada entre filhos de mães pardas em todos os anos da série, com proporções que variaram de 80,3 (2011) a 88,1\% (2010). Quanto ao peso ao nascer, as médias variaram entre o mínimo de 2.050,6 gramas (2008) e máximo de 2.103,6 gramas (2006), com desvios-padrão de 1.049,4 (2011) a 1.108,2 (2009). A cobertura de acompanhamento pré-natal com 07 ou mais consultas variou entre $20,2 \%$ (2011) e $36,9 \%$ (2007). Os maiores percentuais de cobertura de consultas de pré-natal foram identificados na faixa de 4 a 6 consultas, que variou de 34,3\% (2009) a 49,3\% (2010). A proporção de gestantes que não tiveram consulta pré-natal foi elevada, se considerada a série como um todo, mas em 2011, $10,1 \%$ dos óbitos infantis foram relacionados com gestantes que não tiveram acompanhamento.

Tabela 3 - Distribuição dos óbitos infantis por variáveis selecionadas, Piauí, 2006-2011

\begin{tabular}{|l|c|c|c|c|c|c|c|c|c|c|c|c|}
\hline Variáveis & \multicolumn{2}{|c|}{2006} & \multicolumn{2}{|c|}{2007} & \multicolumn{2}{c|}{2008} & \multicolumn{2}{|c|}{2009} & \multicolumn{2}{|c|}{2010} & \multicolumn{2}{|c|}{2011} \\
\hline Escolaridade materna & $\mathrm{n}$ & $\%$ & $\mathrm{n}$ & $\%$ & $\mathrm{n}$ & $\%$ & $\mathrm{n}$ & $\%$ & $\mathrm{n}$ & $\%$ & $\mathrm{n}$ & $\%$ \\
\hline Nenhuma & 28 & 5,7 & 25 & 5,0 & 17 & 3,9 & 17 & 5,6 & 17 & 4,7 & 18 & 4,1 \\
\hline 1 a 4 anos de estudo & 121 & 24,6 & 119 & 23,8 & 112 & 26,0 & 58 & 18,9 & 42 & 11,7 & 49 & 11,2 \\
\hline 5 a 8 anos de estudo & 181 & 36,8 & 184 & 36,9 & 150 & 34,8 & 115 & 37,6 & 109 & 30,4 & 150 & 34,4 \\
\hline 9 a 11 anos de estudo & 119 & 24,2 & 124 & 24,9 & 119 & 27,6 & 98 & 32,0 & 152 & 42,3 & 186 & 42,7 \\
\hline
\end{tabular}




\begin{tabular}{|l|c|c|c|c|c|c|c|c|c|c|c|c|}
\hline 12 de estudo ou mais & 43 & 8,7 & 47 & 9,4 & 33 & 7,7 & 18 & 5,9 & 39 & 10,9 & 33 & 7,6 \\
\hline Raça/cor da mãe & $\mathrm{n}$ & $\%$ & $\mathrm{n}$ & $\%$ & $\mathrm{n}$ & $\%$ & $\mathrm{n}$ & $\%$ & $\mathrm{n}$ & $\%$ & $\mathrm{n}$ & $\%$ \\
\hline Branca & 71 & 14,1 & 82 & 16,1 & 38 & 8,6 & 34 & 12,3 & 33 & 9,1 & 57 & 13,7 \\
\hline Preta & 12 & 2,4 & 16 & 3,1 & 13 & 3,0 & 9 & 3,3 & 8 & 2,2 & 22 & 5,3 \\
\hline Amarela & 3 & 0,6 & 1 & 0,2 & - & - & 3 & 1,1 & 2 & 0,6 & 2 & 0,5 \\
\hline Parda & 416 & 82,7 & 411 & 80,6 & 387 & 88,0 & 229 & 83,3 & 318 & 88,1 & 334 & 80,3 \\
\hline Indígena & 1 & 0,2 & - & - & 2 & 0,4 & - & - & - & - & 1 & 0,2 \\
\hline $\begin{array}{l}\text { Peso ao nascer } \\
\text { gramas) }\end{array}$ & \multicolumn{2}{|c|}{2006} & \multicolumn{2}{|c|}{2007} & 2008 & 2009 & 2010 & \multicolumn{2}{|c|}{2011} \\
\hline Média & 2103,6 & 2099,4 & 2050,6 & 2064,8 & 2063,7 & 2052,5 \\
\hline Desvio padrão & 1086,8 & 1053,0 & 1076,5 & 1108,2 & 1081,7 & \multicolumn{2}{|c|}{1049,4} \\
\hline Mínimo & \multicolumn{2}{|c|}{250,0} & \multicolumn{2}{|c|}{305,0} & \multicolumn{2}{|c|}{165,0} & 100,0 & 232,0 & \multicolumn{2}{|c|}{300,0} \\
\hline Máximo & 6350,0 & 4770,0 & 4710,0 & 4300,0 & 5200,0 & \multicolumn{2}{|c|}{4820,0} \\
\hline Consultas de pré-natal & $\mathrm{n}$ & $\%$ & $\mathrm{n}$ & $\%$ & $\mathrm{n}$ & $\%$ & $\mathrm{n}$ & $\%$ & $\mathrm{n}$ & $\%$ & $\mathrm{~N}$ & $\%$ \\
\hline Nenhuma consulta & 22 & 4,4 & 27 & 5,4 & 10 & 2,3 & 25 & 8,1 & 10 & 2,8 & 44 & 10,1 \\
\hline 1-3 consultas & 100 & 20,1 & 113 & 22,6 & 88 & 20,6 & 88 & 28,5 & 77 & 21,5 & 121 & 27,8 \\
\hline 4-6 consultas & 202 & 40,7 & 176 & 35,1 & 187 & 43,8 & 106 & 34,3 & 177 & 49,3 & 182 & 41,9 \\
\hline 7 e mais consultas & 173 & 34,8 & 185 & 36,9 & 142 & 33,3 & 90 & 29,1 & 95 & 26,4 & 88 & 20,2 \\
\hline
\end{tabular}

Fonte: SINASC/SIM, Ministério da Saúde, 2014.

A tabela 4 mostra que os percentuais de óbitos evitáveis variaram entre os limites de $71,2 \%$ (2006 e 2010) e 77\% (2007), asseverando a perspectiva de que a maior parte das mortes infantis poderia ser evitada por ações relacionadas com o sistema e serviços de saúde.

Tabela 4 - Frequência e distribuição dos óbitos infantis evitáveis na amostra, Piauí, 2006-2011

\begin{tabular}{|l|c|c|c|c|c|c|c|c|c|c|c|c|}
\hline \multirow{2}{*}{ Valores } & \multicolumn{2}{|c|}{2006} & \multicolumn{2}{|c|}{2007} & \multicolumn{2}{|c|}{2008} & \multicolumn{2}{|c|}{2009} & \multicolumn{2}{|c|}{2010} & \multicolumn{2}{|c|}{2011} \\
\cline { 2 - 15 } & $\mathrm{n}$ & $\%$ & $\mathrm{~N}$ & $\%$ & $\mathrm{n}$ & $\%$ & $\mathrm{n}$ & $\%$ & $\mathrm{n}$ & $\%$ & $\mathrm{~N}$ & $\%$ \\
\hline Óbitos não evitáveis & 145 & 28,77 & 149 & 29,04 & 121 & 27,50 & 74 & 23,34 & 105 & 28,85 & 117 & 26,53 \\
\hline Óbitos evitáveis & 359 & 71,2 & 364 & 77,0 & 319 & 72,5 & 243 & 76,7 & 259 & 71,2 & 324 & 73,5 \\
\hline
\end{tabular}

Fonte: SINASC/SIM, Ministério da Saúde, 2014.

A tabela 5 apresenta resultados das proporções dos óbitos evitáveis considerando todo o período, segundo grupos de evitabilidade, mostrando que $72,4 \%$ dos óbitos infantis da amostra eram preveníveis. Não foram identificadas mortes relacionadas com o Grupo 1, reduzíveis por ações de imunoprevenção, mas o Grupo 2, que inclui as causas reduzíveis por adequada atenção à mulher na gestação, apresentou o maior percentual na amostra (30,3\%). Não menos relevantes foram as causas reduzíveis por adequada atenção ao recém-nascido, Grupo 4, e as reduzíveis por ações adequadas de diagnóstico e tratamento, Grupo 5, respectivamente com $24,8 \%$ e 27,5\% entre os óbitos infantis da amostra. Por outro lado, os grupos 3 e 6, respectivamente relacionados com causas reduzíveis por adequada atenção à mulher no parto e por ações adequadas de promoção à saúde, vinculadas a ações adequadas de atenção à saúde, apresentaram bem menores proporções, 
$2,7 \%$ e $1,5 \%$.

Tabela 5 - Distribuição dos óbitos infantis, segundo evitabilidade e grupos de causas reduzíveis, frequência e percentual, Piauí, 2006-2011

\begin{tabular}{lrr} 
Amostra & $\mathrm{n}$ & $\%$ \\
Óbitos totais na amostra & 2.584 & $100 \%$ \\
Óbitos não evitáveis na amostra & 711 & $27,6 \%$ \\
Óbitos evitáveis na amostra & 1.870 & $72,4 \%$ \\
$\quad$ Óbitos evitáveis no Grupo 1 (imunoprevenção) & - & - \\
$\quad$ Óbitos evitáveis no Grupo 2 (atenção à gestação) & 782 & $30,3 \%$ \\
$\quad$ Óbitos evitáveis no Grupo 3 (atenção ao parto) & 337 & $13,0 \%$ \\
$\quad$ Óbitos evitáveis no Grupo 4 (atenção ao neonato) & 640 & $24,8 \%$ \\
$\quad$ Óbitos evitáveis no Grupo 5 (diagnóstico e tratamento) & 71 & $2,8 \%$ \\
$\quad$ Óbitos evitáveis no Grupo 6 (promoção à saúde) & 39 & $1,5 \%$ \\
\hline
\end{tabular}

Fonte: SINASC/SIM, Ministério da Saúde, 2014.

\section{DISCUSSÃO E CONSIDERAÇÕES FINAIS}

A partir da amostra de 2.584 observações, constituída da vinculação de 5.705 registros do SIM a 311.784 registros do SINASC, verificou-se a utilidade do linkage para integrar os dados dos sistemas sobre informações vitais e melhorar a disponibilidade da informação relacionada às variáveis socioeconômicas nesta análise, as quais apresentaram maior lacuna no registro do óbito. Registrou-se que 54,7\% das DO de óbitos infantis não apresentaram o número da DNV, usada como chave primária do linkage neste estudo, expondo uma deficiência da captação de informações e barreira adicional ao monitoramento dos óbitos infantis no Piauí, com base em metodologias de integração de dados dos diferentes sistemas.

Após o linkage obteve-se a redução das lacunas decorrentes das falhas de registro sobre lacunas de informação da amostra. A variável com menor lacuna após a vinculação foi o peso ao nascer, com apenas $0,6 \%$ de falha e a maior falta de informação foi observada na raça/cor da mãe, com $2,9 \%$ de falha. No total, 7,7\% dos registros da amostra ainda apresentaram lacuna de informação após a vinculação dos dados, verificando-se que a informação coletada no momento do registro do nascido vivo foi circunstancialmente menos falha que os registros realizados por ocasião do óbito, especialmente quanto aos dados socioeconômicos da mãe.

Considerando separadamente os dados do SIM e SINASC, a captação da informação sobre a mãe foi mais efetiva no registro do nascimento do que no momento de registro do óbito, ensejando a necessidade de esforços para melhorar a coleta destas informações no momento do preenchimento da DO ou mesmo a adoção da vinculação dos dados de forma sistemática e automática, a partir da mesma chave primária usada neste estudo, a DNV. 
É importante ressaltar que se observou a redução percentual mais acentuada dos óbitos infantis que dos nascimentos (21,9\% versus 9,4\%). Por outro lado, a taxa de mortalidade infantil, que em 2006 era próxima a 20 óbitos por mil nascidos vivos, foi 16,9, em 2011, representando redução de $13,8 \%$. Em termos absolutos, ainda registrou-se um considerável número de crianças que morreram antes de completarem um ano de idade, perfazendo o total de 5.705 mortes.

Quanto ao perfil da mortalidade infantil do Piaú, observou-se o crescimento relativo dos óbitos nas faixas de escolaridade do ensino médio e redução entre as mães que possuíam apenas o ensino fundamental. Noutro lado, a frequência e proporção nos extremos de escolaridade também mostraram redução, embora menor. Estas observações podem representar indícios de mudanças no perfil do óbito infantil no Piauí em relação à escolaridade das mães, mas seria necessário o ajuste estatístico das proporções encontradas aos percentuais desta variável encontrados na população feminina geral.

Quanto à variável de raça/cor da mãe, os filhos de mães pardas permaneceram como o grupo étnico mais afetado nos anos analisados. Os dados não permitiram constatar mudanças em relação à distribuição dos óbitos infantis por raça/cor da mãe, no entanto, deve-se considerar também que no estado há $69,9 \%$ de pardos na sua população gera $1^{14}$, sendo esta característica populacional importante para a interpretação dos achados para esta variável.

Entre os fatores de risco da mortalidade infantil, o peso ao nascer é considerado como importante variável preditiva ${ }^{15}$. Um estudo de coorte nacional incluiu a avaliação de prontuários de 23.940 puérperas identificou entre os potenciais fatores de risco para o óbito neonatal o baixo peso ao nascer, o risco gestacional e as condições gerais do recém nascido ${ }^{15}$. As médias de peso ao nascer entre os óbitos infantis registrados mostraram a ocorrência de médias abaixo de 2.500 gramas, com desvios-padrões pouco superiores a 1.000 gramas, indicando que o óbito infantil afetou mais as crianças com menor peso ao nascer, especialmente abaixo do limiar de 2.500 gramas, corroborando que este é um importante fator de predição para o óbito infantil.

Já o acesso ao acompanhamento pré-natal, representado pelo número de consultas realizadas na gestação, pode refletir também a qualidade dos serviços de Atenção Básica prestados às gestantes, uma vez que reflete o estágio de implantação de protocolos clínicos nacionais de acompanhamento à gestante. Neste estudo, os óbitos infantis foram mais frequentes quando o acompanhamento prénatal teve poucas consultas, sendo as maiores proporções de mortes encontradas entre mães com menor acesso às consultas de pré-natal.

Da análise da evitabilidade dos óbitos infantis do Piauí, considerando os parâmetros da lista de causas de mortes evitáveis por intervenções de saúde ${ }^{3-4}$ para o grupo populacional menor de 05 anos, observou-se que quase $3 / 4$ dos óbitos infantis poderiam ser evitados. Os resultados convergiram com os achados de estudo onde se verificou que a maior parcela das mortes infantis ocorridas entre os anos de 1997 e 2006, no Brasil e região Nordeste, eram preveníveis ${ }^{(4)}$. Na presente análise, a 
proporção dos óbitos evitáveis manteve-se ano a ano em limites elevados, reforçando a necessidade de maior atenção dos sistemas locais para estas mortes, implicando também no reconhecimento de potencial lacuna de qualidade na atenção, corroborando os achados de um estudo com foco na evitabilidade do óbito infantil, realizado em um município de grande porte daquele estado ${ }^{16}$.

Apesar da redução das frequências e taxas do óbito infantil, a proporção de óbitos evitáveis entre menores de um ano de idade não mostrou alteração significativa, o que pode refletir a manutenção de condições socioeconômicas desfavoráveis. No entanto, os óbitos infantis analisados neste estudo foram predominantemente relacionados com causas básicas reduzíveis por ações do setor saúde, refletindo a necessidade de aprimoramento do acesso e qualidade da atenção prestada a essa população, convergindo com os achados de um estudo abrangente voltado para o cenário nacional ${ }^{15}$.

As mortes relacionadas com o Grupo 2 da Lista Brasileira de Mortes Evitáveis ${ }^{3-4}$, que inclui causas reduzíveis por adequada atenção à mulher na gestação, apresentou a maior proporção, e as causas reduzíveis por adequada atenção ao recém-nascido e por ações adequadas de diagnóstico e tratamento também se mostraram relevantes, reforçando a percepção da necessidade de avaliações que se aprofundem na identificação das falhas dos processos de trabalho potencialmente relacionados com as causas determinantes da morte infantil, especialmente no âmbito da Atenção Básica.

Apesar das causas evitáveis por ações relacionadas com o acompanhamento pré-natal aparecerem com a mais alta proporção entre os óbitos infantis analisados, os percentuais de óbitos evitáveis por ações de atenção ao parto e ao neonato, somados alcançaram o percentual de 37,8\%, concorrendo pela maior importância entre as causas evitáveis de óbito infantil no Piauí.

Confirmou-se a necessidade de melhoria no acesso e qualidade do acompanhamento pré-natal e a importância disso para a saúde infantil, dado que os óbitos de crianças cujas mães tiveram acesso a 7 ou mais consultas caiu significativamente entre 2006 e 2011, representando indício de que este aspecto sofreu mudança ao longo do período analisado. No entanto, também cresceram os óbitos relacionados a gestantes que não tiveram nenhuma consulta pré-natal, indicando o aumento proporcional de óbitos infantis entre mulheres que não tiveram acesso ao acompanhamento antenatal.

A principal limitação deste estudo é o seu caráter descritivo, baseado em métodos estatísticos de determinação das médias e proporções das variáveis de interesse no agregado da amostra. Entretanto, diante da pouca disponibilidade de estudos abordando a mortalidade infantil no Piauí, a natureza descritiva desta investigação é etapa necessária ao aprofundamento no tema, recomendando-se o desenvolvimento de novos estudos voltados à identificação das relações mais profundas entre as características aqui analisadas e a mortalidade infantil, tanto em nível individual como populacional.

Em síntese, os resultados deste estudo mostraram que a vinculação dos dados dos sistemas de 
mortalidade e nascidos vivos, SIM e SINASC, pode ajudar a melhorar a qualidade da informação sobre eventos vitais, mediante a adoção de técnicas de linkage para a integração dos dados destes sistemas. No entanto, é imprescindível que a informação usada como chave primária, no caso o número da DNV, esteja disponível no registro do óbito.

Ademais, tendo em vista que algumas variáveis parecem mais propensas a falhas de registro, a depender do momento de coleta da informação, é possível que esforços adicionais concentrados na melhoria da qualidade da informação sobre características socioeconômicas da mãe, níveis de acesso aos cuidados pré-natais e condições gerais do parto e neonato, podem apoiar o enfrentamento da mortalidade infantil no Piauí.

A evolução do perfil da mortalidade infantil no Piauí, nos anos de 2006 a 2011, mostrou que as mudanças verificadas ainda não podem ser consideradas profundas, especialmente quanto aos aspectos socioeconômicos e de acesso aos serviços de saúde, mas estudos adicionais podem ajudar a melhor compreender este fenômeno e suas causas no contexto regional e nacional. Por fim, o alto percentual de mortes infantis evitáveis requer atenção imediata dos governos locais, estadual e nacional, a fim de que a efetividade das ações implementadas possa ser avaliada para obtenção de melhores resultados.

\section{REFERÊNCIAS BIBLIOGRÁFICAS}

1 United Nations. Millennium Development Goals [Internet]. New York: United Nations, 2009. [acesso em 27 dez 2014]. Disponível: http://www.un.org/millenniumgoals/

2 Comissão Nacional sobre Determinantes Sociais da Saúde. Relatório Final, Rio de Janeiro: Fiocruz, 2008.

3 Malta DC, Duarte EC, Almeida MF, Dias MAS, Morais Neto OL, Moura L et al. Lista de causas de mortes evitáveis por intervenções do Sistema Único de Saúde do Brasil. Epidemiol Serv Saúde. 2007; 16(4):233-44.

4 Malta DC, Sardinha LMV, Moura L, Lansky S, Leal MC, Szwarcwald CL et al. Atualização da lista de causas de mortes evitáveis por intervenções do Sistema Único de Saúde do Brasil. Epidemiol Serv Saúde. 2010; 19(3):173-76.

5 Brasil. Ministério da Saúde. Manual dos Comitês de prevenção do óbito infantil e fetal. Brasília: Editora do Ministério da Saúde, 2009.

6 Brasil. Ministério da Saúde. Sistema de Informação sobre Nascidos Vivos- SINASC [Internet]. Rio de Janeiro: Ministério da Saúde, 1994-2012 [acesso em 02 mar 2014]. Disponível: http://tabnet.datasus.gov.br

7 Brasil. Ministério da Saúde. Sistema de Informação sobre Mortalidade - SIM [Internet]. Rio de Janeiro: Ministério da Saúde, 1979-2012 [acesso em 02 mar 2014]. Disponível: http://tabnet. 
datasus.gov.br

8 Barbosa DRM, Almeida MG. Características clínico-epidemiológicas e distribuição espacial da mortalidade infantil no Nordeste do Brasil entre 2008 e 2011. G\&S. 2014; 5(2):569-81.

9 Frias PG, Szwarcwald CL, Souza Junior PRB, Almeida WS, Lira PIC. Correção de informações vitais: estimação da mortalidade infantil, Brasil, 2000-2009. Rev Saúde Pública. 2013; 47(6):1048-58.

10 Brasil. Ministério da Saúde. Rede Interagencial de Informação para a Saúde. Indicadores básicos para a saúde no Brasil-IDB [Internet]. Brasília: Ministério da Saúde; 1990-2011 [acesso em 27 dez 2014]. Disponível: http://tabnet.datasus.gov.br/cgi/idb2012/c01.htm

11 Lima IP, Mota ELA. Avaliação do impacto de uma intervenção para a melhoria da notificação da causa básica de óbitos no estado do Piauí, Brasil. Epidemiol. Serv. Saúde. 2011; 20(3):297-305.

12 Szwarcwald CL. Strategies for improving the monitoring of vital events in Brazil. Int $\mathbf{J}$ Epidemiol. 2008; 7(4):738-44.

13 Santos SLD, Silva ARV, Campelo V, Rodrigues FT, Ribeiro JF. Utilização do método linkage na identificação dos fatores de risco associados à mortalidade infantil: revisão integrativa da literatura. Ciênc. saúde colet. 2014;19(7):2095-104.

14 Instituto Brasileiro de Geografia e Estatística. Censos Demográficos. Sinopse do Censo Demográfico de 2010 [Internet]. [acesso em 30 ago 2014]. Disponível: http://www.ibge.gov.br/ estadosat/temas.php?sigla=pi\&tema $=$ sinopse_censodemog 2010

15 Lansky S, Lima Friche AA, Silva AA, Campos D, Azevedo Bittencourt SD, Carvalho ML et al. Birth in Brazil survey: neonatal mortality, pregnancy and childbirth quality of care. Cad. Saúde Pública. 2014; 30(supl.1): S192-S207.

16 Barreto JOM, Nery IS, Mendes YMMB. Mortalidade Perinatal: uma análise com enfoque na evitabilidade. Cogitare Enferm. 2011; 16(1): 88-95.

Artigo apresentado em 20-11-14

Artigo aprovado em 15-03-15

Artigo publicado no sistema em 22-12-15 